\title{
A LARGE AREA POSITION SENSITIVE X-RAY DETECTOR FOR ASTROPHYSICAL OBSERVATIONS
}

\author{
W.A. MELS, P. LOWES, H.B. BUURMANS, A.C. BRINKMAN, A.P. NABER, A. ROOK \\ and W. ZANDEE
}

Laboratory for Space Research Utrecht, Beneluxlaan 21, 3527 HS Utrecht, The Netherlands

\begin{abstract}
A large area position sensitive X-ray detector has been developed for use in the coded mask imaging X-ray spectrometer (COMIS) aboard the USSR research module KVANT. The module was launched on March 31, 1987. The detector, having a sensitive area of $256 \times 256 \mathrm{~mm}^{2}$, is a sealed multiwire proportional counter filled with Xe gas. It covers the photon energy range 2 to $25 \mathrm{keV}$. At an energy of $6 \mathrm{keV}$ the achieved position resolution is better than $0.6 \mathrm{~mm}$ in the direction parallel to the anode wires. The energy resolution is about $18 \%$ at $6 \mathrm{keV}$.

The paper gives an overview of the development considerations for optimizing performance with respect to linearity and resolution in energy and position response. Results of performance verification are presented.
\end{abstract}

\section{Introduction}

In the last decade coded mask telescopes have been developed for higher energy $\mathrm{X}$-ray and gamma-ray astronomy. Such telescopes enable imaging of hard Xray sources over a large field of view. A coded mask telescope consists of a large area imaging detector and a shadow mask at some distance in front of it. Imaging proportional counters are very suitable detectors for hard X-ray telescopes [1]. The large area IPC described hereafter was designed in 1983 for use in the coded mask imaging spectrometer (COMIS) * [2]. COMIS was launched in March 1987 together with three other X-ray instruments aboard the KVANT module (this module is part of the USSR space station MIR). Several successful observations on X-ray sources have been obtained since switch-on in June.

\section{Description of the COMIS detector}

The detector consists of two separate counters in one sealed stainless steel housing: the actual photon counter and the anticoincidence counter used for background rejection (fig. 1). Both counters are separated by a grid of grounded wires K1. The detector is filled with a 1.1 bar mixture of $95 \%$ xenon and $5 \% \mathrm{CO}_{2}$. The detector

\footnotetext{
* The COMIS project is a collaboration between the Laboratory for Space Research at Utrecht, the Department of Space Research, University of Birmingham, and the Institute for Space Research at Moscow.
}

entrance window consists of $200 \mu \mathrm{m}$ thick beryllium and has an area of $256 \times 256 \mathrm{~mm}^{2}$.

The anode wire grid Al is located half way the photon counter. All anode wires are interconnected except for three wires at both edges of the grid. The anode signal is used to obtain energy information on the X-ray photon and as a trigger for the instrument electronics to start the event processing. The edge wires are used for background rejection, they have increasing diameters to lower the electric field near the wires. At both sides of the anode grid the sense wire grids $\mathrm{S} 1$ and $\mathrm{S} 2$ are located. The wires of $\mathbf{S} 1$ (S2) run perpendicular (parallel) to the anode wires and are used to measure the photon position in the direction parallel (perpendicular) to the anode wires. To minimize disturbing effects on the spatial and spectral response due to the grid edges and the detector walls, the area of the grids $\left(290 \times 290 \mathrm{~mm}^{2}\right)$ has been made larger than the window area. This also enables the rejection of secondary radiation originating from the walls and grid supports. Additional data about the grid system have been summarized in table 1.

For the position readout the wires of a sense grid are interconnected according to the fine-coarse-section readout scheme given in fig. 3 (see also ref. [3]). A sense grid consists of 48 fine sections. Four fine sections make up one coarse section. The fine sections have been interconnected to the corresponding fine sections in the other coarse sections. Each coarse and each fine section readout channel has been connected to a charge sensitive preamplifier followed by an integrating and double differentiating RC network with time constants of 6 and 


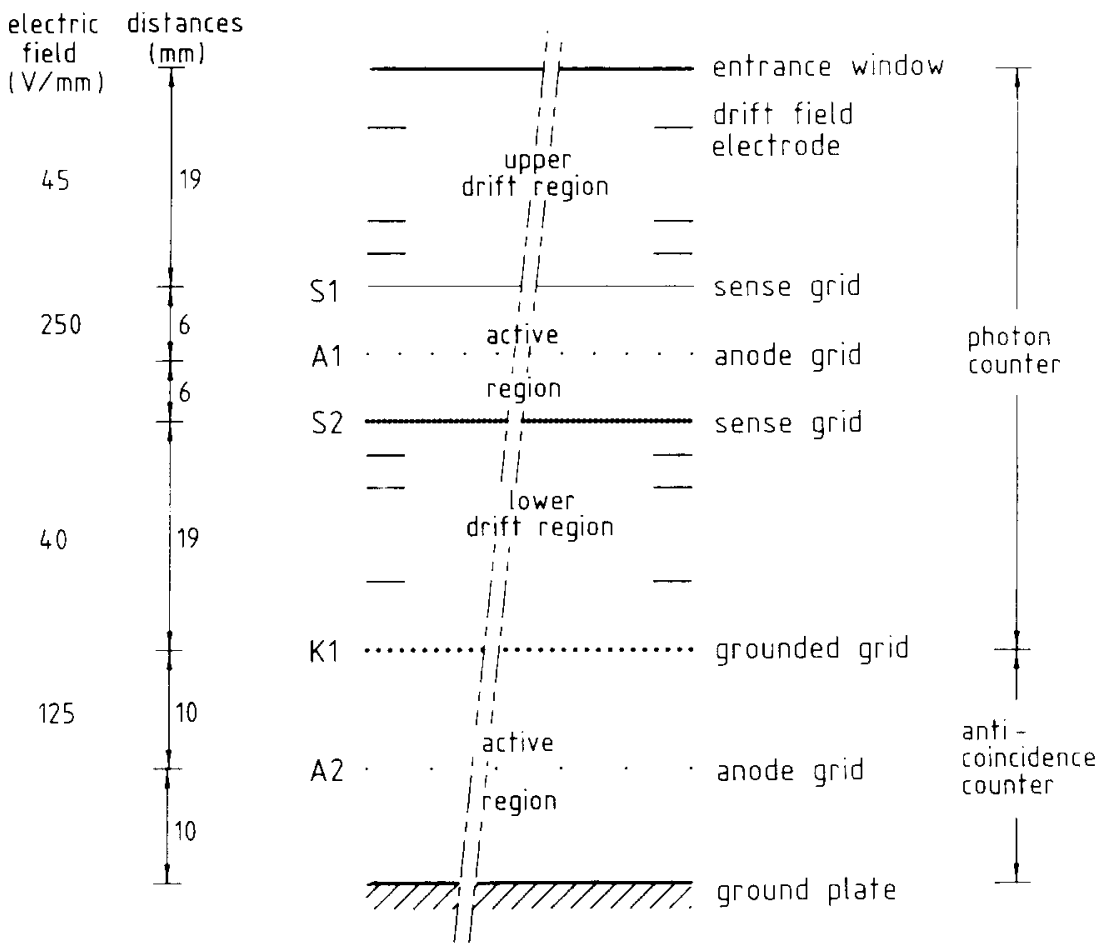

Fig. 1. Schematic diagram of the COMIS detector.

$10 \mu \mathrm{s}$ respectively. Each coarse section channel is equipped with its own discriminator. On the basis of the output pattern of these discriminators it is decided whether the event is legal or not and, if legal, in which coarse section the event has occurred. The fine section channels are read out by a 10-bit ADC. With these data the event position inside the actual coarse section is determined. Validity checks on an event and the position computation are performed by the experiment electronics and computer.

For in-flight calibration purposes, the window support structure contains four ${ }^{55} \mathrm{Fe}$ radioactive sources. Each source produces a collimated $6 \mathrm{keV} \mathrm{X-ray} \mathrm{beam} \mathrm{of}$ $0.2 \mathrm{~mm}$ FWHM and an intensity of about 0.1 photon per second. In addition there is one $22 \mathrm{keV}{ }^{109} \mathrm{Cd}$ source

Table 1

Wire grid date

\begin{tabular}{|c|c|c|c|c|}
\hline & $\mathrm{S} 1 / \mathrm{S} 2$ & $\mathrm{~A} 1$ & A2 & K1 \\
\hline Wire diameter $[\mu \mathrm{m}]$ & 50 & 20 & 20 & 50 \\
\hline Wire pitch [mm] & 0.6 & 3.0 & 6.0 & 1.2 \\
\hline Voltage on wires [V] & 800 & 3400 & 2400 & 0 \\
\hline $\begin{array}{l}\text { Electric field on } \\
\text { surface of wires } \\
{[\mathrm{kV} / \mathrm{mm}]}\end{array}$ & 0.8 & 24.0 & 24.0 & 1.2 \\
\hline Wire material & \multicolumn{4}{|c|}{ Gold-plated tungsten } \\
\hline
\end{tabular}

with an intensity of 0.05 photon per second. Fig. 2 shows a front view of the detector.

\section{Development considerations}

\subsection{Basic approach}

The overall design of the instrument has been strongly influenced by the fact that the instrument has to withstand the demanding environment during launch and has to be able to operate for more than a year in space. In particular, also the deteriorating effects in orbit of gas exposure to high energetic background radiation were taken into account. Therefore emphasis was put on reliability and simplicity. A sealed detector system and a conservative electrical design, the latter with a view to high voltage breakdown risks and aging effects, were chosen.

Since most astrophysical objects of interest for COMIS emit the bulk of their energy in the 2 to $10 \mathrm{keV}$ band, the instrument has been optimized for this range. The design goals of the detector can be summarized as follows:

- sensitive area $500 \mathrm{~cm}^{2}$;

- effective energy band 2-25 keV;

- spatial resolution $\leqq 0.5 \mathrm{~mm}$ in both directions for the energy band $2-10 \mathrm{keV}$ and for X-ray beam 


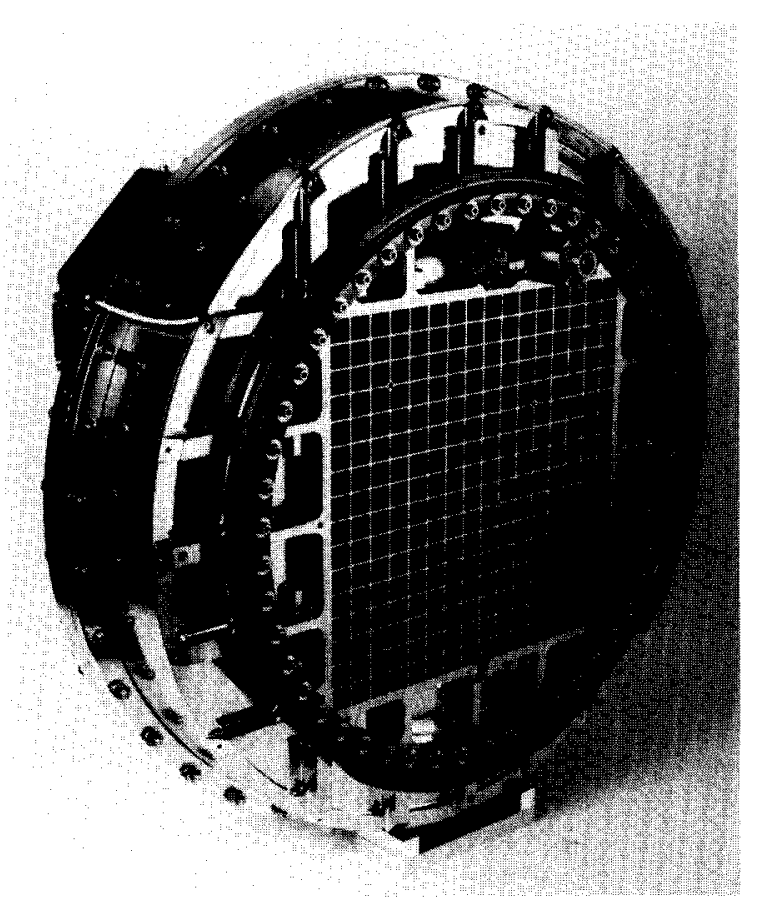

Fig. 2. Front view of the COMIS detector.

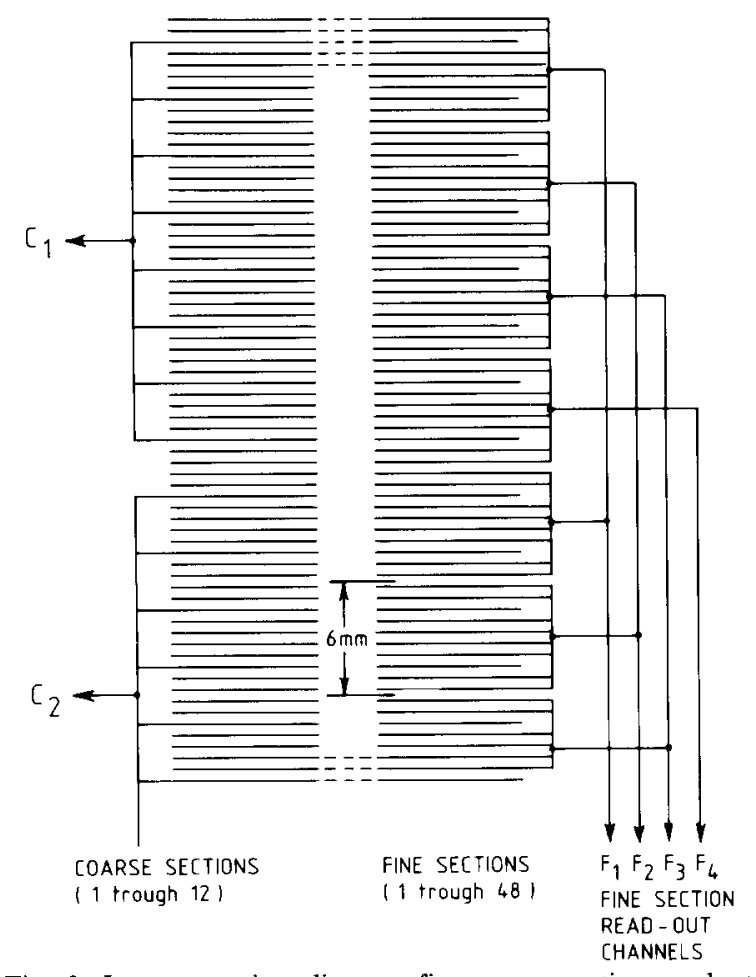

Fig. 3. Interconnection diagram fine-coarse-section readout scheme. inclinations up to $5^{\circ}$ (for energies above $10 \mathrm{keV}$ a spatial resolution worsening to a few $\mathrm{mm}$ is accepted);

- spectral resolution $18 \%$ at $6 \mathrm{keV}$ and better than $20 \%$ at $22 \mathrm{keV}$;

- no performance degradation for count rates up to $1000 \mathrm{cps}$

- optimum background rejection.

To obtain a spread of less than $0.6 \mathrm{~mm}$ ("FWHM") in the position of $10 \mathrm{keV}$ photons for a beam inclination of $5^{\circ}$, the detector has been filled with 1.1 bar xenon gas. $\mathrm{CO}_{2}$ has been selected as a quench gas, since it is a good UV absorber and has a low sensitivity to ageing effects. To obtain the necessary photon counting efficiency up to $25 \mathrm{keV}$ the gas volume is made $50 \mathrm{~mm}$ deep.

The distance between the anode wires is an important parameter. A rather large pitch $(3 \mathrm{~mm})$ was selected for reasons of reliability. A large pitch means a low field strength in the active region (which results in less sensitivity to electrical breakdown) and, in connection with it, a lower voltage on the anode grid. A drawback of a large pitch is the moderate high count rate performance. This is due to the large shaping time constant $(10 \mu \mathrm{s})$ needed to obtain the required spatial resolution and to the long tail on the signals caused by the long drift time of the positive ions to reach the sense grid $(350 \mu \mathrm{s})$.

The COMIS detector operates at a gas gain of 15000 . This results in a $75 \mathrm{fC}$ anode charge for $2 \mathrm{keV}$ (uncorrected for ballistic deficit). At this gain the spectral resolution for photons in the upper part of the energy range is affected by space charge effects as discussed in section 3.3. However, priority has been given to an adequate spatial resolution at $2 \mathrm{keV}$, which demands such a gas gain.

\subsection{Position readout}

The point of absorption of a photon is derived from the center of gravity $Z$ of the charge distribution induced on the sense grids by the avalanche charge. This center is read out using the fine and coarse section signals [4] resulting in a position estimator value. Next this value is translated into the actual photon position by means of a "nonlinearity table". The final spatial resolution depends on the spread in $Z$, the resolution in the readout of the actual $Z$ value and the nonlinearities in the position determination.

The spread in $Z$, in case of normal incidence, is determined by the photoelectron range, the electron diffusion and by xenon fluorescent photons. For the spatial resolution of the COMIS detector, in the energy band of 2 to $10 \mathrm{keV}$, these are relatively minor effects. Fluctuations in the position of the avalanche centroid by UV photon emission, in the active area, may be a significant effect (see section 4.1). 
The resolution in the readout of $Z$ is determined by the sensitivity of the readout scheme and the signal to noise ratio in the electronics. The sensitivity of the fine-coarse-section readout scheme was investigated. To describe the induction distribution the Gatty expression was used [5]. The sensitivity is almost the same for both directions because the shapes of the induction distributions do not differ too much. The sensitivity depends on the width of a fine section and on the number of fine sections in a coarse section. The width of a fine section has to be approximately equal to the distance between the anode and the sense grids, which is $6 \mathrm{~mm}$. We limited the number of fine sections per coarse section to four because the fine section electronics is much more complex than the coarse section electronics chain. In the COMIS detector the capacitance of a fine section channel is $270 \mathrm{pF}$ resulting in a noise of $0.6 \mathrm{pC}$ in the readout electronics of that channel. With this scheme it is possible to achieve the required readout resolution at an acceptable gas gain level and with a reasonable amount of hardware.

The algorithm used in the COMIS instrument for position calculation is a simple linear combination of the charge values measured on the fine sections. With this algorithm the potential sensitivity of the readout scheme is approximated satisfactorily for all values of $Z$. The position estimator is almost linear with $Z$. The estimator is independent of "common mode" fine section signals (crosstalk, pileup, offsets) because the algorithm uses the difference between fine section signals.

In the direction parallel to the anode wires on the average the photon position coincides with $Z$. In the direction perpendicular to the anode wires this relation is very nonlinear due to the fact that the primary electron clouds are smaller than the spacing between the anode wires. In practice the relation between the estimator and the photon position is used and is determined experimentally. The anode wires are arranged with respect to the S2 sense grid such that the nonlinearity in the relationship between the estimator and the event position is repeating every half fine section. As a result this function needs only to be defined for half a fine section interval in order to know the nonlinearity over the whole detector area.

The spatial resolution equals the spread in the estimator multiplied by the differential nonlinearity between the estimator and the actual photon position. The predicted spatial resolution of the COMIS detector, as far as affected by electronic noise only, is shown in fig. 4. As can be seen the resolution in the direction parallel to the anode wires is almost constant, but the resolution in the perpendicular direction just above an anode is two times worse $(0.5 \mathrm{~mm})$ reflecting the differential nonlinearity.

The time constant of the shaping networks in the fine section channels is an important parameter in

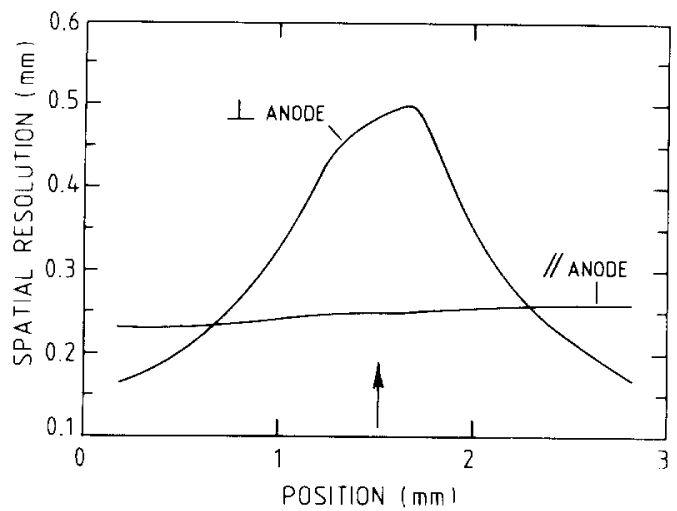

Fig. 4. Predicted spatial resolution for $2 \mathrm{keV} \mathrm{X-rays,} \mathrm{as} \mathrm{far} \mathrm{as}$ affected by electronic noise only, as a function of the photon position over half a fine section interval. The arrow indicates the position of the anode wire.

optimizing the position resolution. The nonlinearity in the direction perpendicular to the anode wires, the thermal noise figure, the ballistic deficit and the sensitivity to pileup and vibration are all affected by the shaping characteristics of the electronics. To minimize the sensitivity to pileup and vibration a double-differentiating RC shaping is used to cut off the lower frequencies as much as possible. The optimum time constant is larger than $10 \mu \mathrm{s}$, however, to limit pileup and vibration effects we decided to use a time constant of $10 \mu \mathrm{s}$.

The instrument is able to determine whether the event occurred above or below the anode grid by comparing the total charge induced on S1 and S2. This information is used to improve the position resolution at higher energies for inclined beams and is also used for background rejection.

\subsection{Energy determination}

In case drift regions in the detector volume are used, the spectral resolution is easily affected [6]. In the COMIS detector electron loss to the window and the sense grids is not significant. It has been found that the spectral resolution of events detected in the drift field region is not degraded by electron attachment as long as the drift voltage is in excess of $100 \mathrm{~V}$ (fig. 5).

A complication, however, arises in case of detection of high energy photons. These photons are detected either in the drift region or in the active region. If the drift voltage is low, the spectral resolution is degraded because the signal response to the "drift region events" is lower than the response to "active region events" (fig. 5). It is unlikely that this effect is caused by loss of electrons in the drift region since the difference disappears at lower gas gain (fig. $5 \mathrm{f}$ ). A possible explanation is that the difference in signal response is caused by the compression of the primary electron clouds of the "drift 

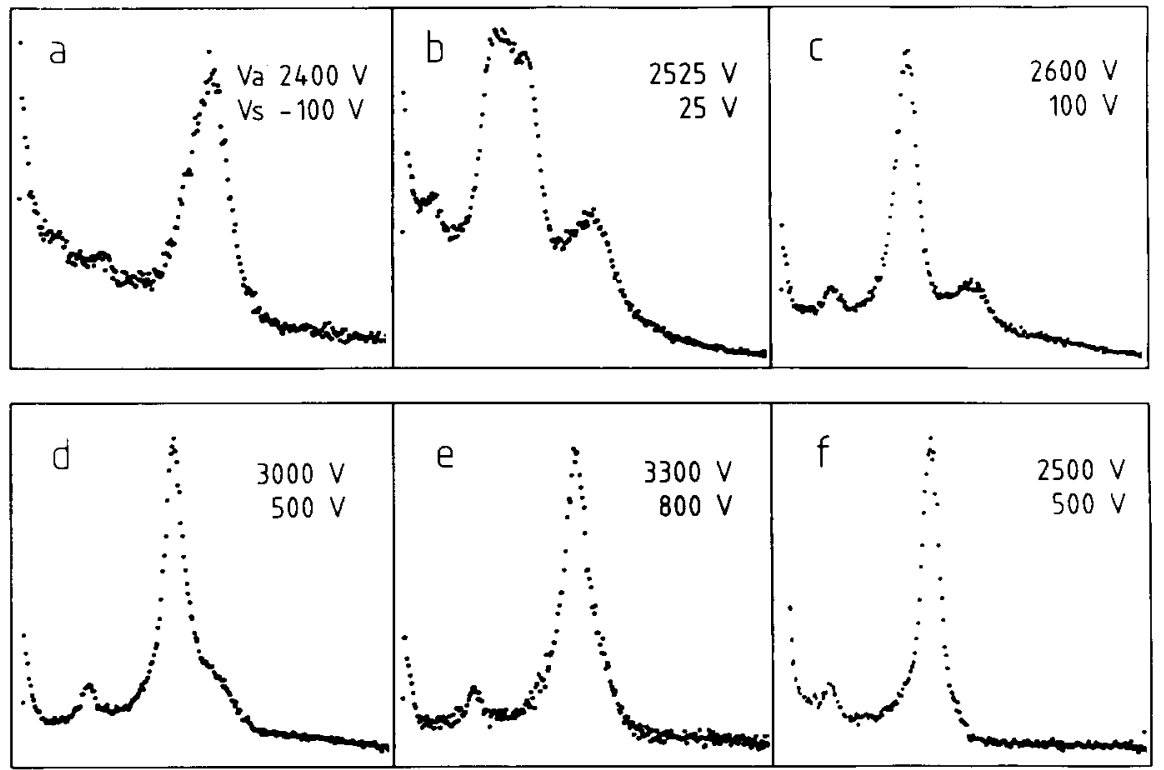

Fig. 5. Spectral response to $22 \mathrm{keV}$ rays $\left({ }^{109} \mathrm{Cd}\right)$ for different electrode voltages. $V_{\mathrm{a}}$ is the voltage on the anode grid, $V_{\mathrm{s}}$ is the voltage on the sense grid. In (a) the drift field is reversed so that only "active region events" are detected. In (b) electron attachment occurs due to a low drift field. In (b), (c) and (d) the spectral resolution is degraded due to a different response to drift and active region events. In (e) and (f) the resolution is better than $20 \%$.

region events" when passing through the sense grid. Such dense clouds may result in avalanches suffering more space charge saturation effects (particularly at higher photon energies) than avalanches of "active region events". The compression of the clouds depends on the drift voltage. Experimentally, it was found that a drift voltage of about $800 \mathrm{~V}$ is needed to obtain a spectral resolution better than $20 \%$ at $22 \mathrm{keV}$.

The effective energy resolution also depends on gain variations across the detector area. Mechanical tolerances are defined such as to keep the gain variations less than $5 \%$. In the direction perpendicular to the anode wires the gain slightly varies with the position relative to the anode wires. The amplitude of this variation increases with increasing avalanche charge, due to space charge effects. In a position sensitive detector in principle these gain variations can be corrected for, if desired.

\subsection{Background rejection}

An important factor in determining the instrument sensitivity is the detector background. A discussion of this topic is beyond the scope of this paper. Certain criteria are applied to discriminate between legal X-ray events and background events. Events with simultaneous response in the anticoincidence counter A2 (cf. fig. 1) or in the edge wires of the anode grid A1 are rejected by hardware. Also events with an equivalent anode signal of more than $25 \mathrm{keV}$ are rejected. The output pattern of the coarse discriminators is inspected by the instrument computer. An event is considered to be illegal if more than two coarse sections are triggered, if two nonconsecutive coarse sections are triggered or if only one coarse section is triggered at the edge of the sense frames. An event with an energy of less than 8 $\mathrm{keV}$, and detected in the lower drift region, is rejected.

\section{Performance}

\subsection{Spatial response function}

The spatial response in the direction parallel to the anode wires obtained with a pinhole beam having a diameter of $0.1 \mathrm{~mm}$, and expressed as a function of the measured anode charge, is shown in fig. 6 . The measured resolution diverges from what one would expect purely on the basis of signal to noise considerations and the beam width.

Data shown in fig. 6 are calculated by an off-line processor using the data acquired from the ADC and the coarse section discriminators directly. In the following, the presented data have been calculated by the instrument processor. The photon position is expressed in pixels with a size of $0.375 \times 0.375 \mathrm{~mm}^{2}$.

The integral nonlinearity over the full detector area in both directions, as derived from the instrument response to uniform illumination, is less than 0.4 pixel. 


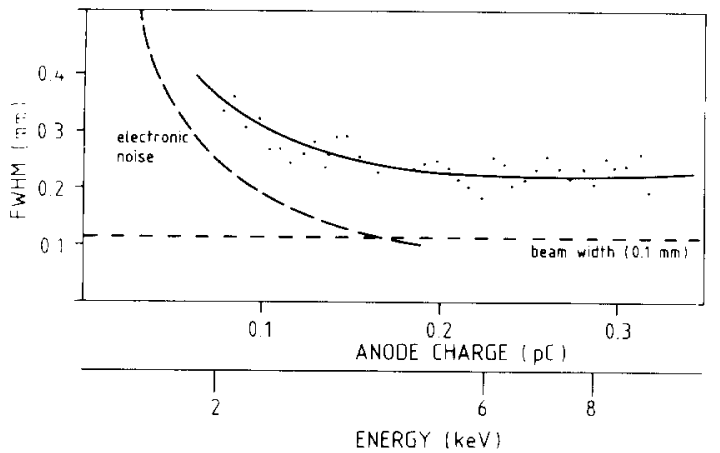

Fig. 6. Resolution in spatial response (in the direction parallel to the anode wires) to a $0.1 \mathrm{~mm}$ pinhole flux as a function of the measured anode charge. The pinhole is illuminated by a continuum of X-rays.
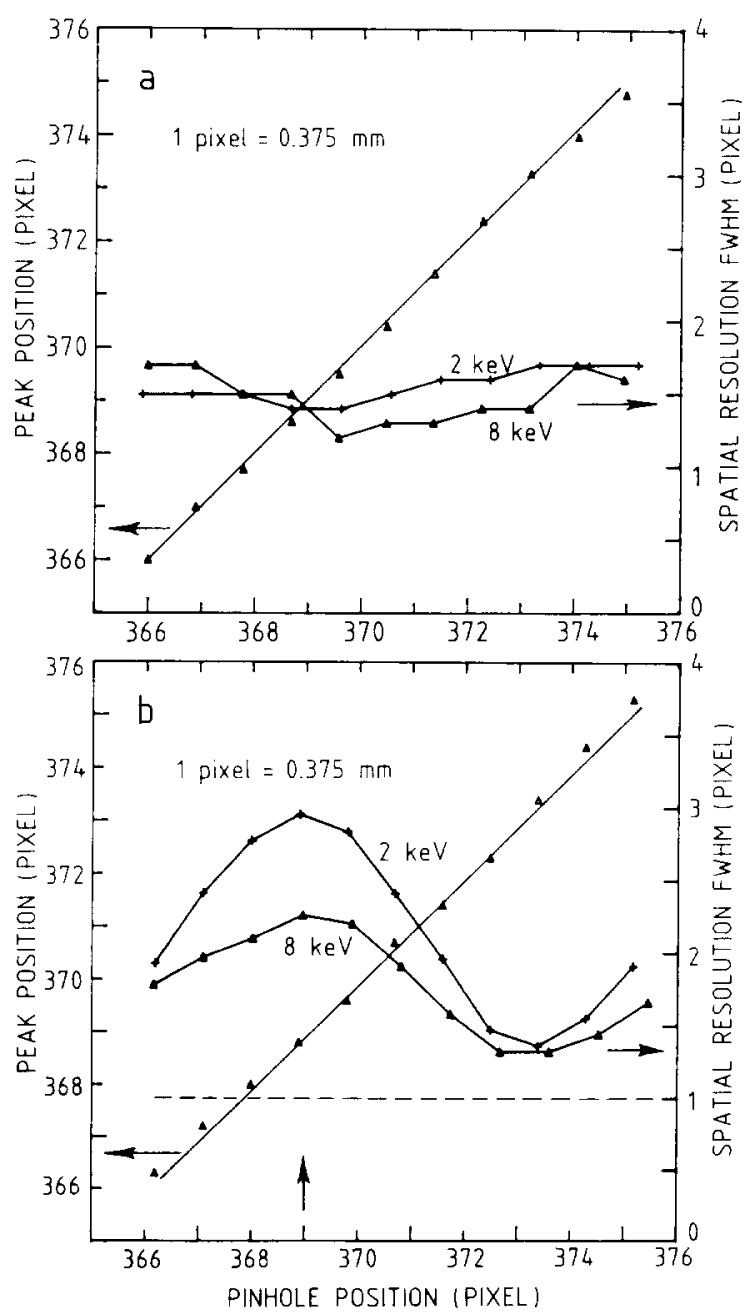

Fig. 7. Spatial resolution and peak positions as a function of the position of $0.1 \mathrm{~mm}$ pinholes for 2 and $8 \mathrm{keV} \mathrm{X}$-rays. In (a) the curves for the direction parallel to the anode wires are given, in (b) the curves for the other direction. The arrow indicates the position of an anode wire.
The response to a set of equidistant pinholes (with a diameter of $0.1 \mathrm{~mm}$ ) for 2 and $8 \mathrm{keV} \mathrm{X}$-rays is measured in both directions. In fig. 7 peak positions as a function of the pinhole position are shown. As can be seen the nonlinearity is less than 0.2 pixel in both directions. Fig. 7 also shows the spatial resolution as a function of the pinhole position. In the direction parallel to the anode wires the resolution is mainly limited by the pixel size. In the direction perpendicular to the anode wires, above an anode wire the resolution for $2 \mathrm{keV}$ X-rays $(2.9$ pixel) is two times worse than the resolution taking into account the electronic noise only (see fig. 4 ).

The charge in the avalanche varies from $75 \mathrm{fC}$ at 2 $\mathrm{keV}$ to more than $750 \mathrm{fC}$ at $25 \mathrm{keV}$. It is reported $[7,8]$ that for these charge levels the position of the avalanche centroid (and $Z$ ) is affected by UV photons emitted by excited Xe gas atoms. Possibly this effect accounts for the difference between the measured spectral resolution and the values predicted on the basis of electronic noise and $\mathrm{X}$-ray beam width.

By means of the in-flight calibration sources the spatial response can be studied in orbit as a function of different background rates. The peak positions remain constant within a fraction of a pixel for background rates up to $1000 \mathrm{rps}$. The resolution remains in agreement with the data shown in fig. 7 .

\subsection{Energy response function}

Fig. 8 shows the response to 6 and $22 \mathrm{keV} \mathrm{X-rays} \mathrm{for}$ a uniform detector illumination. The resolution becomes $18 \%$ and $15 \%$ respectively. This result confirms that corrections for gain variations across the detector sensitive area are unnecessary.

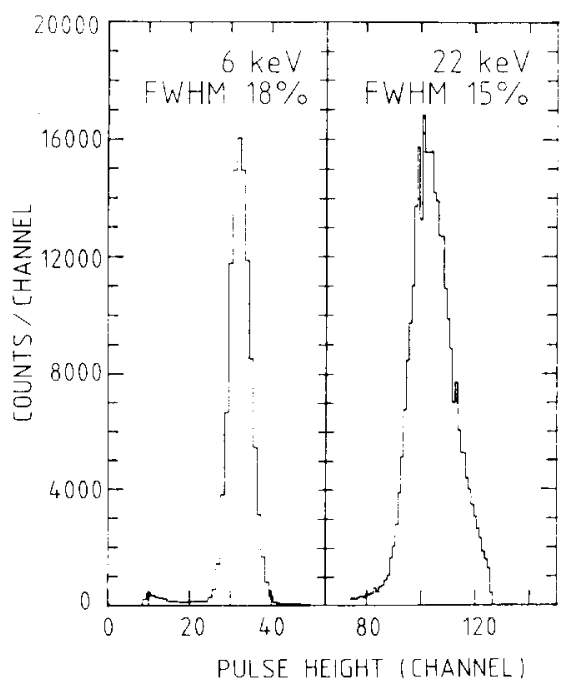

Fig. 8. Spectral response to uniform illumination by 6 and 22 keV X-rays. 


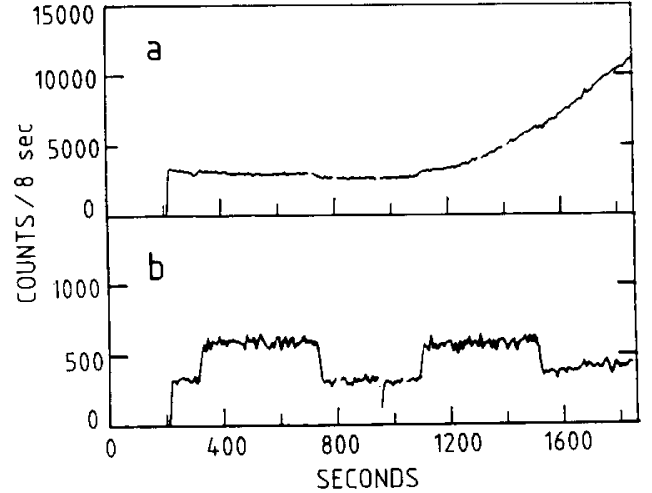

Fig. 9. Event rate as a function of time as measured in orbit, (a) event rate without background rejection, (b) event rate after background rejection by hardware and software criteria.

The nonproportionality of the detector for $22 \mathrm{keV}$ photons with respect to $6 \mathrm{keV}$ photons is $18 \%$.

\subsection{Background rejection}

In fig. 9 the detector count rate, as measured in-flight, is shown as a function of time. The variations in the corrected event rate are due to pointing manoeuvres of the spacecraft with respect to an observed stellar X-ray source. The rejection efficiency for the full background measured by the instrument at that time is found to be better than $98.5 \%$.

\section{Conclusions and future developments}

The measured performance of the COMIS detector with regard to the spatial and spectral response is in agreement with the requirements except for the spatial resolution in the direction perpendicular to the anode wires. In this direction the spatial resolution for $2 \mathrm{keV}$ varies from 0.5 to $1.0 \mathrm{~mm}$, the average resolution is 0.8 $\mathrm{mm}$. At $6 \mathrm{keV}$ the average resolution is $0.7 \mathrm{~mm}$ for count rates up to $1000 \mathrm{cps}$.

Future coded mask instruments to be developed at our laboratory will have a larger field of view than the COMIS instrument. For those instruments we then need detectors with a higher gas pressure, and a better high count rate performance than the COMIS detector. We are presently investigating the influence of the anode pitch and the anode grid distance on the detector performance with respect to spatial and spectral resolution and their impact on system reliability.

\section{References}

[1] G. Charpak, SPIE Proc. X-ray Instrumentation in Astronomy, vol. 597 (1985) p. 170.

[2] A.C. Brinkman et al., Proc. Int. Workshop, Rome (1983) p. 263.

[3] Van Beek et al., IEEE Trans. Nucl. Sci. NS-31 (1984) 45.

[4] G. Charpak et al., Nucl. Instr. and Meth. 148 (1978) 471.

[5] E. Gatti et al., Nucl. Instr. and Meth. 163 (1979) 83.

[6] D.G. Simons et al., SPIE Proc. X-ray Instrumentation in Astronomy, vol. 597 (1985) p. 190.

[7] H.D. Thomas and M.J.L. Turner, Nucl. Instr. and Meth. 221 (1984) 175.

[8] J. Fischer et al., Nucl. Instr. and Meth. A252 (1986) 239 